\title{
Model uncertainties of local-thermodynamic-equilibrium K-shell spectroscopy
}

T. Nagayama, J. E. Bailey, G. Loisel, G. A. Rochau, and S. B. Hansen

Sandia National Laboratories, Albuquerque, New Mexico 87185, USA

C. Blancard, Ph. Cosse, G. Faussurier, F. Gilleron, and J.-C. Pain

Commissarat à I'Énergie Atomique (CEA) et aux Énergies Alternatives, F-91297 Arpajon,

France

J. Colgan, C. J. Fontes, D. P. Kilcrease, and M. E. Sherrill

Los Alamos National Laboratory, Los Alamos, New Mexico, 87545, USA

H. K. Chung

International Atomic Energy Agency, A-1400 Vienna, Austria

R. Florido

Departamento de Física, Universidad de Las Palmas de Gran Canaria, 35017 Las Palmas de

Gran Canaria, Spain

I. E. Golovkin and J. J. MacFarlane

Prism Computational Sciences, Madison, Wisconsin 53703, USA

C. A. Iglesias

Lawrence Livermore National Laboratory, Livermore, California 94550, USA

R. W. Lee

Institute for Material Dynamics at Extreme Conditions, University of California, Berkeley,

California, 94720, USA

R. C. Mancini

University of Nevada, Reno, Nevada 89557, USA

(Dated: May 23, 2016)

Local-thermodynamic-equilibrium (LTE) K-shell spectroscopy is a common tool to diagnose electron density, $n_{e}$, and electron temperature, $T_{e}$, of high-energy-density (HED) plasmas.

Knowing the accuracy of such diagnostics is important to provide quantitative conclusions of 
many HED-plasma research efforts. For example, Fe opacities were recently measured at multiple conditions at the Sandia National Laboratories Z machine [J. E. Bailey et al, Nature 517, 56 (2015)], showing significant disagreement with modeled opacities. Since the plasma conditions were measured using K-shell spectroscopy of tracer Mg [T. Nagayama et al, Physics of Plasmas 21, 056502 (2014)], one concern is the accuracy of the inferred Fe conditions. In this article, we investigate the K-shell spectroscopy model uncertainties by analyzing the Mg spectra computed with 11 different models at the same conditions. We find that the inferred conditions differ by $\pm 20-30 \%$ in $n_{e}$ and $\pm 2-4 \%$ in $T_{e}$ depending on the choice of spectral model. Also, we find that half of the $T_{e}$ uncertainty comes from $n_{e}$ uncertainty. To refine the accuracy of the $\mathrm{K}$-shell spectroscopy, it is important to scrutinize and experimentally validate line-shape theory. We investigate the impact of the inferred $n_{e}$ and $T_{e}$ model uncertainty on the Fe opacity measurements. Its impact is small and does not explain the reported discrepancies.

\section{INTRODUCTION}

In high-energy-density (HED) plasmas, emergent K-shell spectral lines are sensitive to electron density, $n_{e}$, and electron temperature, $T_{e}$, of the source plasma [1-5]. The spectral line shapes strongly depend on $n_{e}$ via Stark line broadening. The ratios of spectral lines from adjacent charge states are very sensitive to $T_{e}$. K-shell spectroscopy is considered well understood and is a common tool to diagnose $n_{e}$ and $T_{e}$ of HED plasmas.

We recently measured Fe opacity at multiple conditions relevant to the solar interior and revealed 30-400 \% disagreement with modeled Fe opacities [6]. The $n_{e}$ and $T_{e}$ of those measurements were determined with $\mathrm{K}$-shell spectroscopy of tracer $\mathrm{Mg}$ [5]. One may question the accuracy of these inferred conditions. While the reported measurement uncertainties included experiment-to-experiment reproducibility and fit uncertainties due to noise, they did not include all the uncertainties associated with the analysis method itself. For example, the noise in the data can affect not only the fit but also some intermediate processing steps such as the continuum-baseline determination discussed in Ref. [5]. Also, the measured absorption lines are saturated to some extent due to the instrumental broadening. To minimize those concerns, we avoided heavily saturated lines in the analysis and accounted for the instrumental broadening effects on the modeled spectra. However, the instrumental broadening does slightly reduce the diagnostic sensitivity, and this contribution should be assessed. Perhaps the most important 
uncertainty neglected in Ref. [5] is that associated with potential inaccuracy of the spectral model. Validating spectral models requires dedicated experiments and is beyond the scope of this work, but we can at least investigate how much difference in $n_{e}$ and $T_{e}$ arises if different spectral models are used in the interpretation.

In this article, we quantify the uncertainties of inferred $n_{e}$ and $T_{e}$ due to the analysis method and the choice of spectral model through analyses of synthetic data. We first verify that the analysis method accurately infers conditions within $2 \sigma$ of the fit uncertainty even when accounting for noise and the saturation due to the instrumental broadening. Then, we investigate the K-shell spectroscopy model uncertainty by analyzing Mg K-shell spectra calculated with 11 different models at the conditions relevant to the Fe opacity experiments [5]. We find that the inferred conditions can vary by $\pm 20-30 \%$ in $n_{e}$ and $\pm 2-4 \%$ in $T_{e}$ depending on the choice of spectral model. This significant $n_{e}$ discrepancy originates from differences in the line-shape calculations in the spectral models. Since $n_{e}$ affects the charge-state distributions, this $\pm 20-30 \%$ $n_{e}$ uncertainty is also responsible for half of the $T_{e}$ uncertainty. It is crucial to use accurate line-shape models for reliable K-shell spectroscopic diagnosis. Theoretical scrutiny and experimental validation of these line-shape models are essential for refining our understanding of HED plasmas. We further investigate the impact of these $n_{e}$ and $T_{e}$ uncertainties on the reported Fe opacity discrepancies and confirm they do not explain the major discrepancy.

The paper is organized as follows. Our investigation method and the synthetic data creation method are discussed in Sec. II. We investigate the potential inaccuracy of our analysis method due to noise, instrumental broadening, and continuum-baseline determination in Sec. III. Then, we numerically investigate the inferred-condition dependence on the spectral model in Sec. IV. Potential sources of the discrepancies are discussed in Sec. V. The impact of the $n_{e}$ and $T_{e}$ uncertainty due to the spectral model dependence on the recent Fe opacity measurement is discussed in Sec. VI, and the conclusions are given in Sec. VII.

\section{SYNTHETIC DATA CREATION}

To create various synthetic data, Mg K-shell opacity spectra are computed with 8 different models: ABAKO [7], ATOMIC [8, 9], FLYCHK[10], OPAL [11], OPAS [12, 13], PrismSPECT [14], SCO-RCG [15], and SCRAM [16]. Multiple calculations are submitted from some models with different line-shape calculation options or with different atomic-structure calculation options. As a result, there are a total of 11 different calculations for each condition. 
The spectra are computed at 1) $n_{e}=0.9 \times 10^{22} \mathrm{~cm}^{-3}$ and $T_{e}=165 \mathrm{eV}$ and 2) $n_{e}=4.0 \times 10^{22} \mathrm{~cm}^{-3}$ and $T_{e}=195 \mathrm{eV}$, which are similar to the conditions achieved by the recent Fe opacity measurements $[5,6]$. We remind the reader that we observed agreement between modeled and measured Fe opacities at the lower $n_{e}$ and $T_{e}$ conditions [17], while significant disagreement was found at the higher $n_{e}$ and $T_{e}$ case [6].

Synthetic data are computed from calculated opacities as follows. First, opacity spectra are converted to transmission spectra by:

$$
T_{v}^{\text {modeled }}=\exp \left(-\rho L \kappa_{v}^{\text {modeled }}\right)
$$

where $\kappa_{v}$ is the calculated opacity in $\mathrm{cm}^{2} / \mathrm{g}$, and $\rho L$ is typical $\mathrm{Mg}$ areal density used in our experiments. We use $3.0 \times 10^{-5}$ and $4.9 \times 10^{-5} \mathrm{~g} / \mathrm{cm}^{2}$ for each case. Second, we convolve transmission with instrumental broadening:

$$
T_{v}^{\text {instrum }}=\int g\left(v-v^{\prime}\right) T_{v^{\prime}}^{\text {modeled }} d v^{\prime}
$$

where $g\left(v-v^{\prime}\right)$ is the measured spectral resolution [18] of our spectrometers [4]. Then, a few-percent Poisson noise (i.e., typical in our measurements) is added to the spectra. We extract $n_{e}$ and $T_{e}$ from these synthetic data using the analysis method discussed in Ref. [5], permitting us to investigate the accuracy of our analysis method.

\section{ANALYSIS METHOD ACCURACY}

There are several concerns in the accuracy of the analysis discussed in Ref. [5]. First, bound-bound ( $b-b)$ line transmission spectra were extracted from the measured absorption spectra by defining a smooth continuum baseline from the measured absorption spectra and dividing the spectra by this baseline [5]. The motivation behind this was that the spectral range where Mg K-shell lines appear also had significant Fe bound-free (b-f) continuum absorption. Since it was impossible to separate $\mathrm{Mg}$ b-f from the Fe b-f absorption and $n_{e}$ and $T_{e}$ sensitivities exist in the Mg b-b lines, we extracted Mg b-b line transmission spectra for $n_{e}$ and $T_{e}$ analysis. Since Mg b-b lines were well isolated, it was rather straightforward to define the baseline. However, it is always a concern how the noise in the data can affect the baseline determination and the analysis results. Second, for efficient analysis, we construct a Mg opacity database using PrismSPECT [14] with detailed line shapes computed with MERL [19, 20]. A genetic algorithm followed by a Levenberg-Marquardt non-linear least-squares minimization method [21] interpolates the database and searches for the optimal $n_{e}$ and $T_{e}$ to reproduce the data. A concern 
is whether or not the analysis based on the database with linear interpolation provides sufficiently accurate results. Finally, we would like to know how accurately we can infer $n_{e}$ and $T_{e}$ under line saturations due to instrumental broadening. To minimize errors associated with this complexity, we measured spectra with an instrument with high resolving power $(E / \Delta E \approx 900)$, use only weaker lines in the analysis, and take into account the instrumental broadening on the modeled transmission spectra. However, it is still not clear how accurately $n_{e}$ can be inferred from altered line shapes because density sensitivity must be somewhat reduced.

We can test potential analysis inaccuracy due to the above limitations by comparing the target conditions with the conditions inferred from the synthetic data, which are directly computed with PrismSPECT with the MERL line shapes. Red lines in Fig. 1 are the b-b line transmission spectra extracted from the PrismSPECT synthetic data. They are computed at (a) $n_{e}$ $=0.9 \times 10^{22} \mathrm{~cm}^{-3}$ and $T_{e}=165 \mathrm{eV}$ and (b) $n_{e}=4.0 \times 10^{22} \mathrm{~cm}^{-3}$ and $T_{e}=195 \mathrm{eV}$. To minimize the saturation effects on the analysis, we analyze weaker lines whose optical depths are $\$ 1.0$ : $\mathrm{Ly} \alpha, \operatorname{He} \gamma, \operatorname{He} \delta$, and $\operatorname{Ly} \beta$ at the lower $n_{e}$ and $T_{e}$ case, and $\operatorname{He} \gamma$ and $\operatorname{Ly} \beta$ at the higher $n_{e}$ and $T_{e}$ case. Fig. 2 shows the results of the chi-square fit of the diagnostic lines. The reduced $\chi^{2}$ is close to unity, quantitatively confirming the goodness of the fit. The conditions inferred by the fit-optimization are (a) $n_{e}=(0.95 \pm 0.04) \times 10^{22} \mathrm{~cm}^{-3}$ and $T_{e}=166 \pm 1 \mathrm{eV}$ and (b) $n_{e}=(4.2 \pm 0.1)$ $\times 10^{22} \mathrm{~cm}^{-3}$ and $T_{e}=195 \pm 1 \mathrm{eV}$. These agree with the target conditions that are used to create the synthetic data within $2 \sigma$ and $1 \sigma$ of the fit uncertainty, respectively. This confirms that the analysis method using the selected lines still infers conditions accurately within $\pm 5 \%$ in $n_{e}$ and $\pm 1 \%$ in $T_{e}$ even under the noise and the small line saturation due to the instrumental broadening.

\section{SPECTRAL-MODEL UNCERTAINTY}

For each condition, 11 synthetic spectra computed with different models (Sec. II) are analyzed using the Mg opacity database constructed with PrismSPECT and MERL and the methods discussed in Sec. III. Since the same analysis method is employed, the standard deviation in the inferred conditions represents the uncertainties due to the spectral-model differences.

Fig. 3 shows $n_{e}$ (left) and $T_{e}$ (right) inferred from synthetic data computed with different models, plotted in percent difference from the mean. The diagnostic uncertainties due to the spectral models are (a) $\Delta n_{e}= \pm 19 \%$ and $\Delta T_{e}= \pm 2.0 \%$ at the lower $n_{e}$ and $T_{e}$ case and (b) $\Delta n_{e}=$ 
$\pm 24 \%$ and $\Delta T_{e}= \pm 3.5 \%$ at the higher $n_{e}$ and $T_{e}$ case, which are more significant than those due to the analysis method (Sec. III).

There is a correlation between the inferred $n_{e}$ and $T_{e}$; when a model inferred higher $n_{e}$, it also inferred higher $T_{e}$. This indicates that some of the $T_{e}$ uncertainty originates from the $n_{e}$ uncertainty. The spectra constrain line shapes and relative populations. When higher $n_{e}$ is inferred, the model has to infer higher $T_{e}$ to reproduce the relative population in the synthetic data. To check this hypothesis, we repeat the analyses by fixing the $n_{e}$ to the mean of the inferred $n_{e}$. Then, the standard deviations of the inferred $T_{e}$ shrink from \pm 2.0 to $\pm 1.0 \%$ and from \pm 3.5 to $\pm 1.6 \%$ at each condition, respectively. This means that half of the $\Delta T_{e}$ originates from more-serious disagreement in $n_{e}$, which must be produced by the differences in the line-shape calculations employed in the spectral models.

\section{COMPARISON OF MERL AND TOTAL}

While there are 11 different spectral models in this investigation, they use either Voigt approximations or a standard Stark-line-shape model for their spectral line shapes. Three out of 11 spectral models use Voigt profiles with parameters calculated differently for each model. One model determines its Lorentzian widths by natural and collisional broadening and its Gaussian widths by thermal and Stark broadening [22, 23]. Two models determine the Voigt parameters from their own empirical formulae based on fits to a standard Stark-line-shape model [19, 20]. The rest of the models employed one of standard Stark-line-shape models; two models use MERL [19, 20], five models use TOTAL [24], and one model uses SCO-RCG [25].

In Fig. 3, the density inferred from the synthetic spectra with the same line-shape models are not identical. For example, model 3 through 7 all use TOTAL, but the inferred densities vary by $\pm 10 \%$ and $\pm 16 \%$ for Fig. 3(a) and (b), respectively, and are beyond the fit uncertainties. This variation comes from the differences in the versions of TOTAL, atomic data, ion-microfield calculation model, input of microfield model such as $T_{e}$, mean charge, and composition. While scrutinizing these details is interesting, we focus on the bigger disagreement between MERL and TOTAL (and SCO-RCG) to get better insight on the main source of discrepancy and its impact on our Fe opacity measurements.

In the investigation shown in Fig. 3, the three standard line-shape models, MERL, TOTAL, and SCO-RCG all assume static ions (see Fig. 4 to confirm negligible ion-dynamic effects). The main differences are their calculations in the electron broadening and the basis set 
used to approximate the perturbation on the upper states (Table I). We note that these are the options used in this investigation and do not necessarily mean the only options available for the models. As one can see in Fig. 3, SCO-RCG shows comparable results to TOTAL due to its similar treatment of strong collisions in the electron-broadening calculation [26] and their use of a non-extended basis set. Thus, in this section, we pick MERL and TOTAL, and scrutinize them for potential sources of the diagnostic discrepancies.

MERL [19, 20] and TOTAL [24] are widely used in the HED-plasma community and considered to be reasonably accurate and consistent with each other. However, as shown in Fig. 3, when we use the same tool to analyze the spectra computed with MERL and TOTAL, we infer 30-60\% higher density from those with MERL (i.e., model 1 and 2). This indicates that line shapes computed with MERL are broader than those with TOTAL.

To better understand the potential source of density discrepancies, $\mathrm{Mg} \mathrm{He} \gamma$ line shapes computed with TOTAL (red) and MERL (blue) are compared in Fig. 4. There are two fundamental differences between these two models. First, MERL computes this He $\gamma$ with an extended basis set [20]. Basically, when a line shape is computed, the atomic states perturbed by charged particles are approximated by a linear combination of unperturbed atomic states. At lower density, the perturbation of the upper states of the transition (e.g., $1 \mathrm{~s} 4 \mathrm{p}{ }^{1} P_{1}$ and $1 \mathrm{~s} 4 \mathrm{p}{ }^{3} P_{1}$ for $\mathrm{He} \gamma$ ) can be well approximated with a linear combination of the unperturbed states in the $n=$ 4 manifold (i.e., 1s $4 \mathrm{~s}, 1 \mathrm{~s} 4 \mathrm{p}, 1 \mathrm{~s} 4 \mathrm{~d}$, and 1s $4 \mathrm{f}$ for $\mathrm{He} \gamma$ ). However, as density increases, the mixing with unperturbed states in the next manifold (i.e., 1s $5 \mathrm{~s}, 1 \mathrm{~s} 5 \mathrm{p}, 1 \mathrm{~s} 5 \mathrm{~d}, 1 \mathrm{~s} 5 \mathrm{f}$, and $1 \mathrm{~s} 5 \mathrm{~g}$ for He$\gamma$ ) becomes important [20]. The dashed line is the He $\gamma$ line shape computed with MERL while neglecting the $n=5$ manifold, and the significant difference from the solid line indicates its importance.

Another major difference is the dipole electron-broadening operator, or G-function. MERL employs the G-function computed from the Gaunt factor [27], while TOTAL computes it from the dielectric constants [24]. It was recently pointed out that the electron broadening employed in TOTAL could be more accurate than that of MERL due to more appropriate treatment of the strong-collision contributions (see Ref [26] for a detailed discussion of the two codes). His points were supported by measured neutral hydrogen Balmer line shapes [29]. However, the reported agreement does not gurantee the calculation accuracy for charged radiatiors because the use of dielectric constants neglects the impact of nuclear charge on the 
perturbing electrons. Since $\mathrm{Mg} \mathrm{He}$ - and H-like ions are highly charged radiators, its true accuracy remains in question. We also note that a significant disagreement was previously reported between measured and modeled electron broadening for charged radiators, and the source of the discrepancy has not been identified yet [30,31]. Hence, further theoretical investigations and benchmark experiments are needed to refine our understanding of the line-broadening calculations and reliability of K-shell spectroscopy. The workshop of Spectral Line Shapes in Plasmas is held every two years to scrutinize the differences in the theoretical line-shape model details and their impact on the calculated line shapes [32-35]. Such workshops are an appropriate place for further scrutiny on the theoretical details and their impact on $n_{e}$ diagnostics.

\section{IMPACT ON THE RECENT FE OPACITY MEASUREMENTS}

In Sec. IV, we find the $n_{e}$ and $T_{e}$ inferred with K-shell spectroscopy could be different by $\pm 20-40 \%$ in $n_{e}$ and $2-4 \%$ in $T_{e}$ depending on the choice of spectral models. In Sec. V, we find that the main source of uncertainty is the difference in the line-shape models employed in the spectral models and, thus, is systematic. Since the plasma conditions for the recent Fe opacity measurement relied on the MERL line-shape model $[5,6]$, it is interesting to know how the modeled/measured Fe opacity discrepancy would improve/deteriorate with the $n_{e}$ inferred from a different line-shape model (e.g., TOTAL). As an illustration, we picked the conditions, $n_{e}=4.0 \times$ $10^{22} \mathrm{~cm}^{-3}$ and $T_{e}=195 \mathrm{eV}$, where modeled/measured Fe opacity disagree by $30-400 \%$ [6].

While the standard deviation of inferred $n_{e}$ and $T_{e}$ are $\pm 20-40 \%$ and 2-4\%, respectively, the systematic difference between models employing different line-shape models is even larger. For example, for Fig. 3(b), we infer $67 \%$ higher $n_{e}$ and $5 \%$ higher $T_{e}$ from the synthetic data created with MERL than those with TOTAL [Fig. 3 (b)]. This implies that the MERL line shapes are broader than the TOTAL ones for the diagnostic lines. If the TOTAL line shapes turned out to be accurate, the conditions that are inferred with MERL would be incorrect and underestimated. One would need roughly a $67 \%$ increase in $n_{e}$ to reproduce the measured line width using this narrower TOTAL line shape and a $5 \%$ increase in $T_{e}$ to compensate for the density change and recover the measured charge states.

Fig. 5 compares the measured Fe opacity from Ref. [6] and the Fe opacities computed with SCRAM at the two conditions: $n_{e}^{\mathrm{MERL}}=4 \times 10^{22} \mathrm{~cm}^{-3}$ and $T_{e}^{\mathrm{MERL}}=195 \mathrm{eV}$ and $n_{e}^{\text {TOTAL }}=6.7 \times 10^{22} \mathrm{~cm}^{-3}$ and $T_{e}^{\text {TOTAL }}=205 \mathrm{eV}$. The first conditions are those inferred with 
PrismSPECT with MERL [5]. The second conditions are the conditions that a spectral model with TOTAL would infer and are approximated by increasing $n_{e}^{\mathrm{MERL}}$ and $T_{e}^{\mathrm{MERL}}$ by $67 \%$ and $5 \%$, respectively. The instrumental broadening effects on the modeled spectra are taken into account by computing transmission spectra, applying instrumental broadening, and converting them back to opacity spectra. While it is interesting to see similar comparisons for all the spectral models in Ref [6], one model is sufficient to show the impact of model uncertainty on the reported discrepancy; we pick SCRAM as an example. The SCRAM spectrum at $n_{e}^{\text {TOTAL }}$ and $T_{e}^{\mathrm{TOTAL}}$ is very similar to that at $n_{e}^{\mathrm{MERL}}$ and $T_{e}^{\mathrm{MERL}}$ except for the line width. This is because not only $n_{e}$ but also $T_{e}$ are increased to reproduce the measured $\mathrm{Mg}$ charge-state distribution, and this ends up reproducing the Fe charge-state distribution as well. In reality, the true conditions are likely to be somewhere between these two conditions, because MERL line shapes are too broad due to the lack of appropriate treatment of strong collisions [26] and TOTAL line shapes are too narrow due to the non-extended basis set (Sec. V). It is unlikely that inferred-condition inaccuracy is the main source of the reported Fe opacity discrepancy.

\section{CONCLUSIONS}

We investigated the model uncertainty of local-thermodynamic-equilibrium K-shell spectroscopy and found that the inferred conditions could vary $\pm 20-30 \%$ in $n_{e}$ and $\pm 2-4 \%$ in $T_{e}$ due to the choice of the spectral model. While the case studied focuses on its application to recent Fe opacity measurements [6], we expect similar impact for other applications. A major source of the K-shell spectroscopy uncertainty is the uncertainty in the Stark-line-shape calculations. While the quoted uncertainties are often sufficient for many applications, better diagnostic accuracy is desired. To further improve the reliability of the K-shell spectroscopy, it is important to re-scrutinize the line-shape models both theoretically and experimentally. We investigate the impact of this potential systematic uncertainty on the recent Fe opacity measurement. Its impact is small and does not explain the major discrepancy reported.

\section{ACKNOWLEDGEMENT}

We thank the Z-facility teams for invaluable and dedicated technical assistance. T. N. thanks R. E. Falcon for his careful reading and refinement of the manuscript. Sandia is a multiprogram laboratory operated by Sandia Corporation, a Lockheed Martin Company, for the United States Department of Energy under contract DE-AC04-94AL85000. The Los Alamos 
National Laboratory is operated by Los Alamos National Security, LLC for the NNSA of the U.S. DOE under Contract No. DE-AC5206NA25396. R. F. is partially supported by EUROfusion Consortium, Task Agreement WPENR Enabling Research IFE, Project No. AWP15-ENR-01/CEA-02.

[1] H. Griem, Principles of Plasma Spectroscopy, Cambridge Monographs on Plasma Physics (Cambridge University Press, 2005).

[2] H. R. Griem, Physics of Fluids B: Plasma Physics 4, 2346 (1992).

[3] C. J. Keane, B. A. Hammel, D. R. Kania, J. D. Kilkenny, R. W. Lee, A. L. Osterheld, L. J.

Suter, R. C. Mancini, C. F. Hooper, and N. D. Delamater, Physics of Fluids B: Plasma Physics 5, 3328 (1993).

[4] J. E. Bailey, G. A. Rochau, R. C. Mancini, C. A. Iglesias, J. J. MacFarlane, I. E. Golovkin, J.

C. Pain, F. Gilleron, C. Blancard, P. Cosse, G. Faussurier, G. A. Chandler, T. J. Nash, D. S.

Nielsen, and P. W. Lake, Rev. Sci. Instrum. 79, 113104 (2008).

[5] T. Nagayama, J. E. Bailey, G. Loisel, S. B. Hansen, G. A. Rochau, R. C. Mancini, J. J.

MacFarlane, and I. Golovkin, Phys. Plasmas 21, 056502 (2014).

[6] J. E. Bailey, T. Nagayama, G. P. Loisel, G. A. Rochau, C. Blancard, J. Colgan, P. Cosse, G.

Faussurier, C. J. Fontes, F. Gilleron, I. Golovkin, S. B. Hansen, C. A. Iglesias, D. P. Kilcrease, J. J. MacFarlane, R. C. Mancini, S. N. Nahar, C. Orban, J. C. Pain, A. K. Pradhan, M. Sherrill, and B. G. Wilson, Nature 517, 56 (2015).

[7] R. Florido, R. Rodríguez, J. M. Gil, J. G. Rubiano, P. Martel, E. Mínguez, and R. C. Mancini, Phys. Rev. E 80, 056402 (2009).

[8] P. Hakel, M. E. Sherrill, S. Mazevet, J. Abdallah, J. Colgan, D. P. Kilcrease, N. H. Magee, C. J. Fontes, and H. L. Zhang, J. Quant. Spectrosc. Radiat. Transfer 99, 265 (2006).

[9] C. J. Fontes, H. L. Zhang, J. A. Jr, R. E. H. Clark, D. P. Kilcrease, J. Colgan, R. T.

Cunningham, P. Hakel, N. H. Magee, and M. E. Sherrill, J. Phys. B: At. Mol. Opt. Phys. 48, 144014 (2015).

[10] H. K. Chung, M. H. Chen, W. L. Morgan, Y. Ralchenko, and R. W. Lee, High Energ. Dens. Phys. 1, 3 (2005).

[11] C. A. Iglesias and F. J. Rogers, Astrophys. J. 371, 408 (1991).

[12] C. Blancard, P. Cossé, and G. Faussurier, Astrophys. J. 745, 10 (2012). 
[13] G. Mondet, C. Blancard, P. Cossé, and G. Faussurier, ApJS 220, 1 (2015).

[14] J. J. MacFarlane, I. E. Golovkin, P. R. Woodruff, D. R. Welch, B. V. Oliver, T. A. Mehlhorn, and R. B. Campbell, in Inertial Fusion and Science Applications, edited by B. A. Hammel, Amer. Nucl. Soc. (Proc. Inertial Fusion and . . ., 2004) p. 457.

[15] J.-C. Pain and F. Gilleron, High Energ. Dens. Phys. 15, 30 (2015).

[16] S. Hansen, J. Bauche, C. Bauche-Arnoult, and M. F. Gu, High Energ. Dens. Phys. 3, 109 (2007).

[17] J. E. Bailey, G. A. Rochau, C. A. Iglesias, J. J. Abdallah, J. J. MacFarlane, I. Golovkin, P. Wang, R. C. Mancini, P. W. Lake, T. C. Moore, M. Bump, O. Garcia, and S. Mazevet, Phys. Rev. Lett. 99, 265002 (2007).

[18] G. Loisel, J. E. Bailey, G. A. Rochau, G. S. Dunham, L. B. Nielsen-Weber, and C. R. Ball, Rev. Sci. Instrum. 83, 10E133 (2012).

[19] L. Woltz and C. Hooper, Phys. Rev. A 38, 4766 (1988).

[20] R. C. Mancini, D. P. Kilcrease, L. A. Woltz, and C. F. Hooper Jr., Comput. Phys. Commun. 63, 314 (1991).

[21] T. Nagayama, R. C. Mancini, R. Florido, D. Mayes, R. Tommasini, J. A. Koch, J. A.

Delettrez, S. P. Regan, and V. A. Smalyuk, Phys. Plasmas 19, 082705 (2012).

[22] E. Stambulchik and Y. Maron, J. Phys. B: At. Mol. Opt. Phys. 41, 095703 (2008).

[23] E. Stambulchik and Y. Maron, in The 17th International Conference on Atomic Processes in Plasmas (AIP, 2012) pp. 203-209.

[24] R. W. Lee, J. Quant. Spectrosc. Radiat. Transfer 40, 561 (1988).

[25] J.-C. Pain, F. Gilleron, and D. Gilles, arXiv, arXiv:1512.00603 (2015), 1512.00603.

[26] C. A. Iglesias, High Energ. Dens. Phys. 18, 14 (2016).

[27] J. T. O’Brien and C. F. Hooper Jr., J. Quant. Spectrosc. Radiat. Transfer 14, 479 (1974).

[28] H. Griem, M. Blaha, and P. Kepple, Phys. Rev. A 19, 2421 (1979).

[29] W. L. Wiese, D. E. Kelleher, and D. R. Paquette, Phys. Rev. A 6, 1132 (1972).

[30] S. Glenzer, N. Uzelac, and H. J. Kunze, Phys. Rev. A 45, 8795 (1992).

[31] Y. V. Ralchenko, H. Griem, and I. Bray, J. Quant. Spectrosc. Radiat. Transfer 81, 371 (2003).

[32] E. Stambulchik, High Energ. Dens. Phys. 9, 528 (2013). 
[33] S. Ferri, A. Calisti, C. Mossé, J. Rosato, B. Talin, S. Alexiou, M. Gigosos, M. González, D. González-Herrero, N. Lara, T. Gomez, C. Iglesias, S. Lorenzen, R. Mancini, and E. Stambulchik, Atoms 2, 299 (2014).

[34] M. Koubiti, M. Goto, S. Ferri, S. Hansen, and E. Stambulchik, Atoms 2, 319 (2014).

[35] S. Alexiou, M. Dimitrijević, S. Sahal-Brechot, E. Stambulchik, B. Duan, D.

González-Herrero, and M. Gigosos, Atoms 2, 157 (2014).

[36] D. Boercker, C. Iglesias, and J. Dufty, Phys. Rev. A 36, 2254 (1987).

FIG. 1. Spectra computed with the database at the inferred conditions (blue) successfully reproduce the synthetic data (red) created (a) at $n_{e}=0.9 \times 10^{22} \mathrm{~cm}^{-3}$ and $T_{e}=165 \mathrm{eV}$ and (b) at $n_{e}$ $=4.0 \times 10^{22} \mathrm{~cm}^{-3}$ and $T_{e}=195 \mathrm{eV}$.

FIG. 2. Blow-ups of Fig. 1 for $\mathrm{He} \gamma$ and $\mathrm{Ly} \beta$. The red and blue are synthetic data and the fits, respectively. Reduced $\chi^{2}$ is close to unity, indicating good fits. Inferred conditions agree with the target conditions within $2 \sigma$ of the measurement uncertainty due to noise.

FIG. 3. $n_{e}$ and $T_{e}$ inferred from synthetic data created with 11 different models (a) at $n_{e}=0.9 \times$ $10^{22} \mathrm{~cm}^{-3}$ and $T_{e}=165 \mathrm{eV}$ and (b) at $n_{e}=4.0 \times 10^{22} \mathrm{~cm}^{-3}$ and $T_{e}=195 \mathrm{eV}$ in percent difference from their mean. The dashed lines indicate the standard deviation of the inferred conditions, which are indicated by $\Delta n_{e}$ and $\Delta T_{e}$.

FIG. 4. Mg He $\gamma$ computed with TOTAL (red) and MERL (blue) at $n_{e}=4.0 \times 10^{22} \mathrm{~cm}^{-3}$ and $T_{e}=$ $195 \mathrm{eV}$. The MERL line shape is computed with (solid) and without (dashed) the $n=5$ manifold, indicating the importance of the extended basis set. The green curve is the MERL line shape with the extended basis set and with ion-dynamic effects [36], which is hidden underneath the solid blue line and indicates its negligible impact at these conditions.

FIG. 5. Measured Fe opacity from Ref. [6] and Fe opacities computed with SCRAM at (red) $n_{e}=$ $4.0 \times 10^{22} \mathrm{~cm}^{-3}$ and $T_{e}=195 \mathrm{eV}$ and (green) $n_{e}=6.7 \times 10^{22} \mathrm{~cm}^{-3}$ and $T_{e}=205 \mathrm{eV}$. The first condition is inferred with MERL line shapes, and the second is what would be inferred with TOTAL. The observed discrepancy are not explained by $n_{e}$ and $T_{e}$ uncertainty due to the choice of spectral model.

TABLE I. Detail summary of MERL, TOTAL, and ST line-models electron broadening and state basis set. All these models are used with the option of static-ion approximation. 


\begin{tabular}{|l|l|l|l|}
\hline & $\begin{array}{l}\text { Ion } \\
\text { microfield }\end{array}$ & Electron broadening & $\begin{array}{l}\text { Upper state } \\
\text { basis }\end{array}$ \\
\hline MERL[19, 20] & APEX & Gaunt factor [27] & $n_{u}, n_{u}+1$ \\
\hline TOTAL[24] & APEX & Dielectric constant [24] & $n_{u}$ \\
\hline SCO-RCG [25] & Potekhin & Semi-classical [28] & $n_{u}$ \\
\hline
\end{tabular}


Figure 1

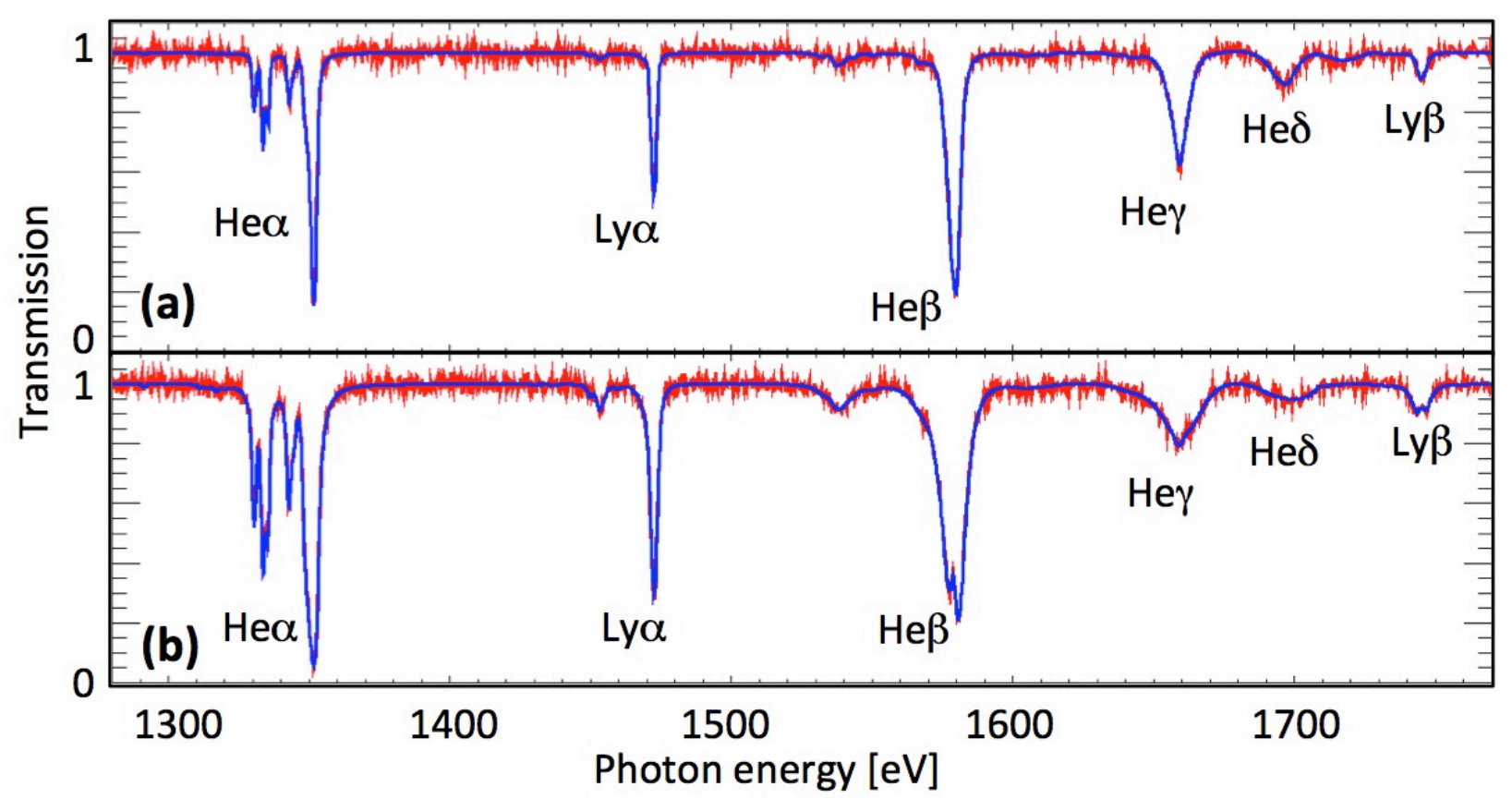


Figure 2
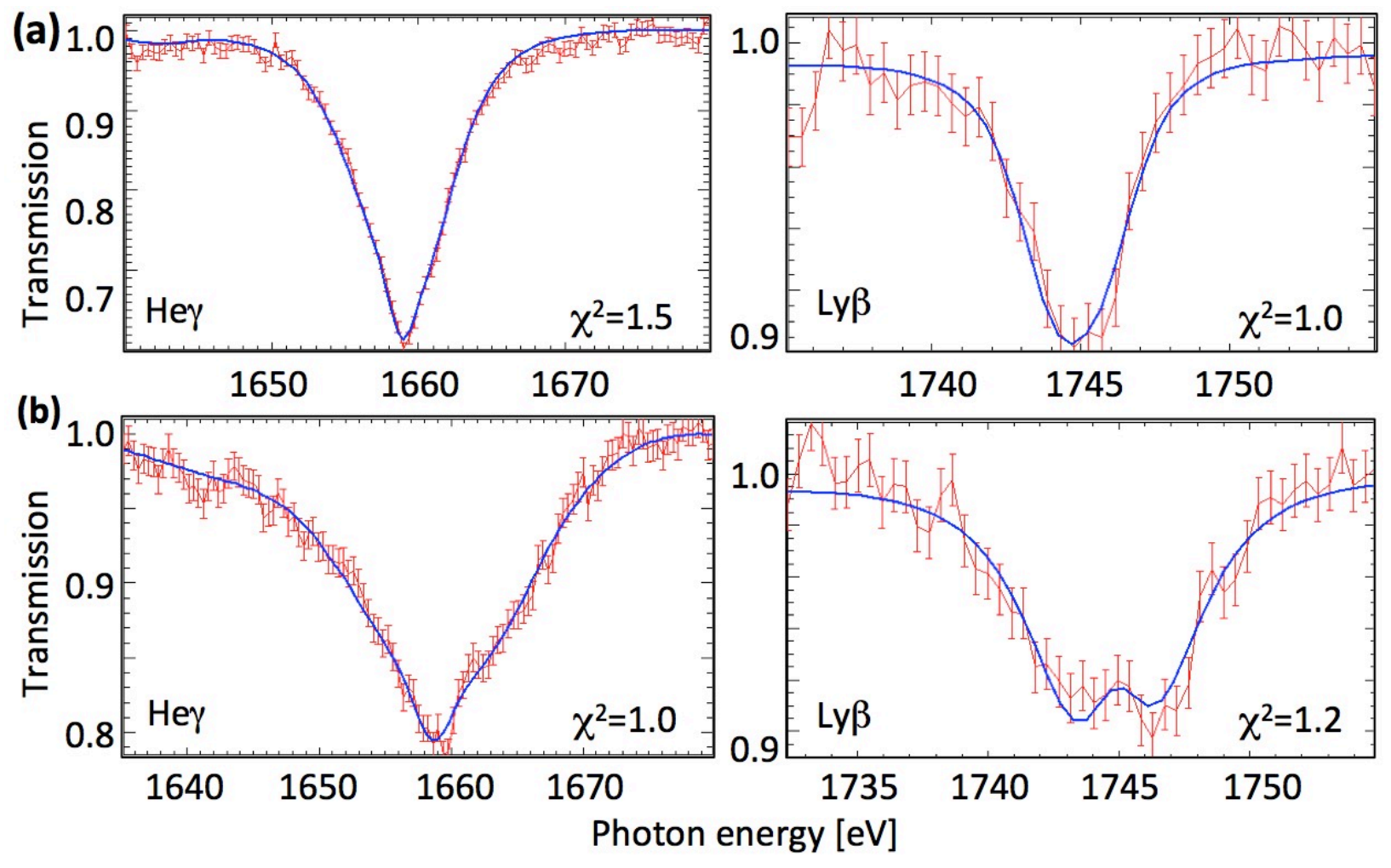
Figure 3
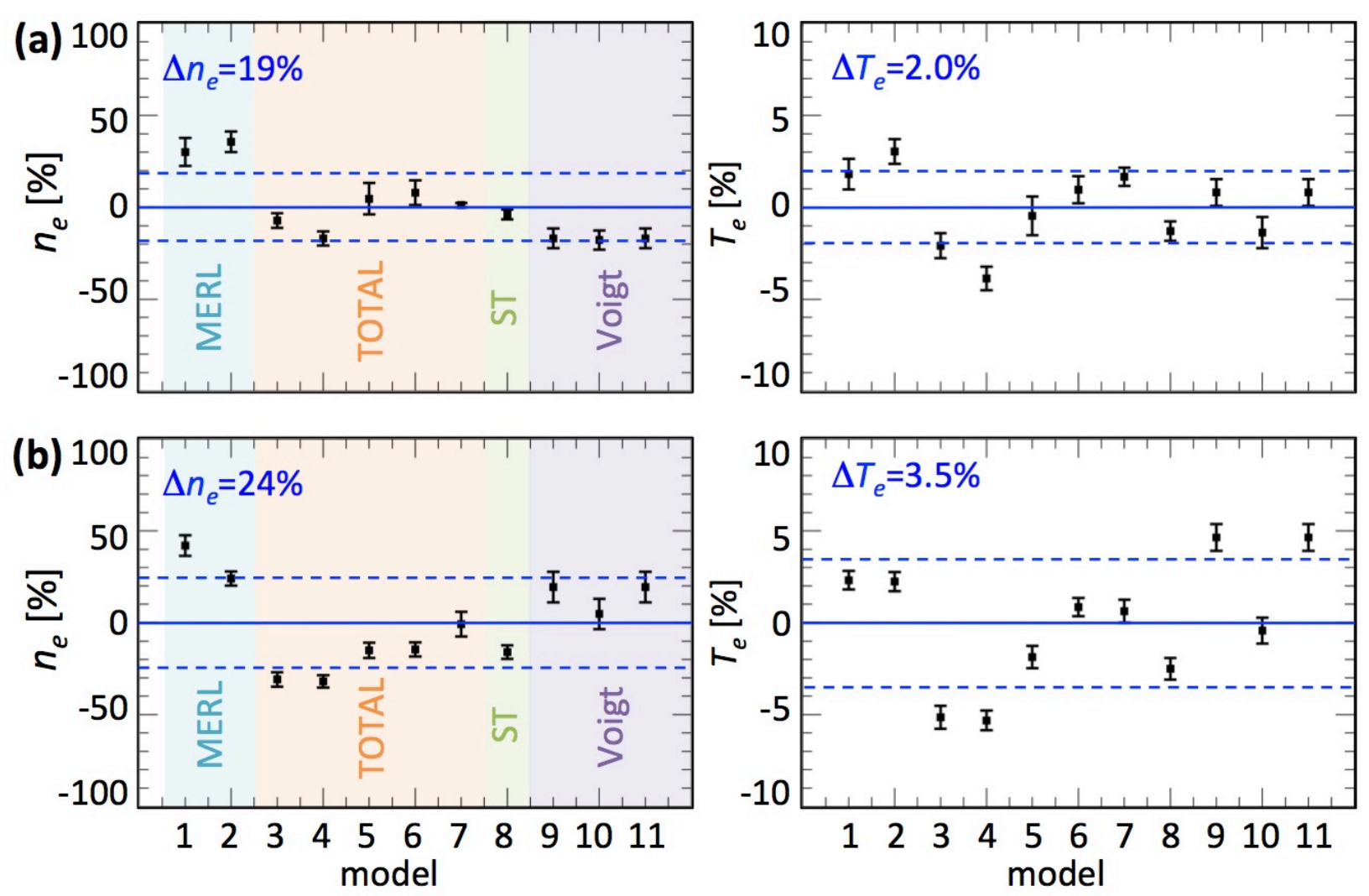
Figure 4

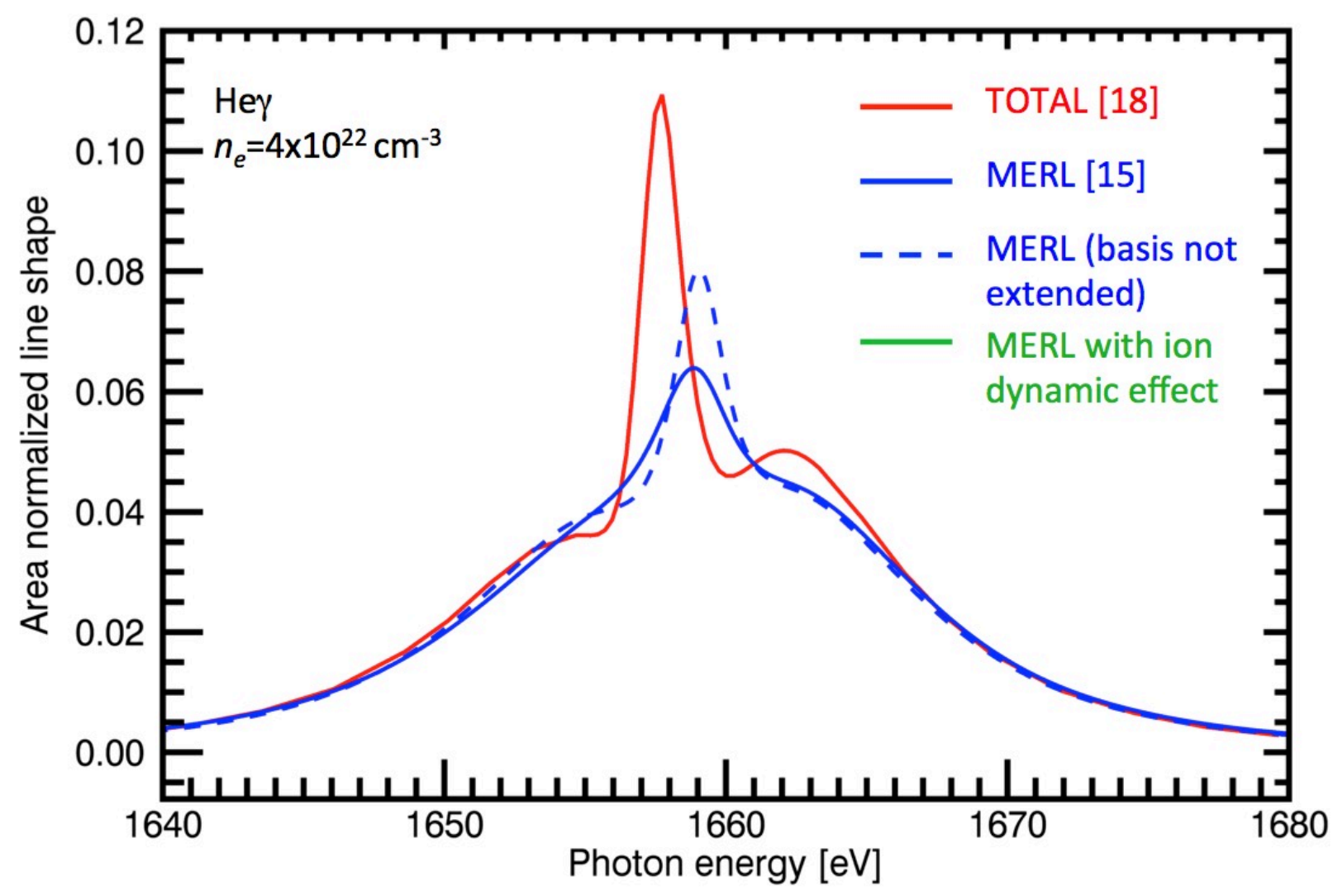


Figure 5

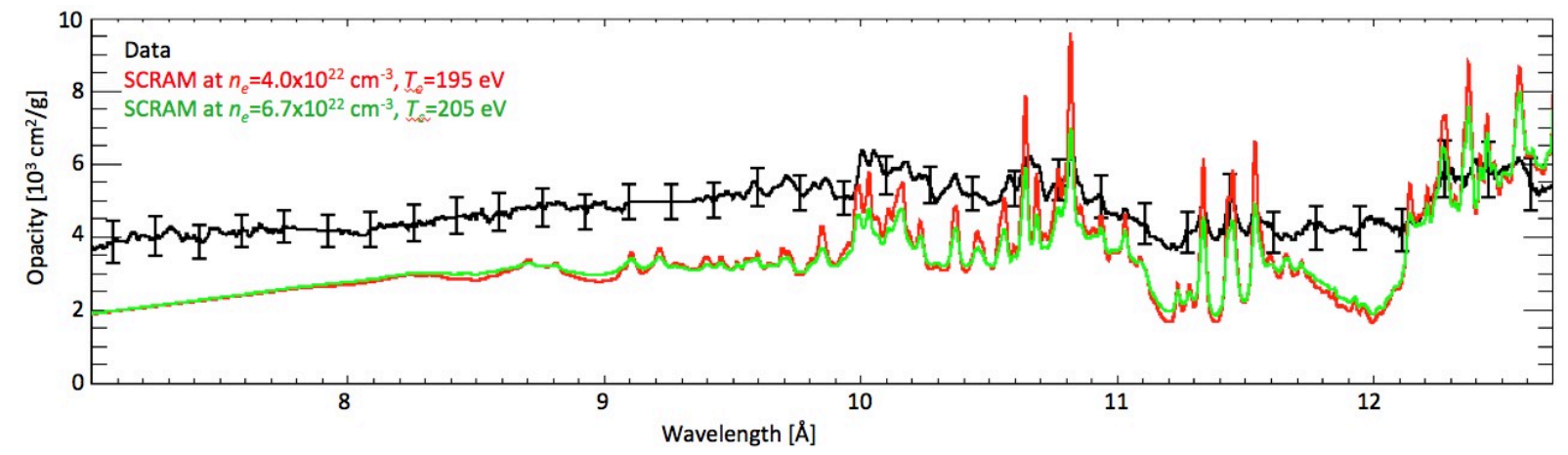

